\title{
THE MID-WINTER ASSEMBLAGE AND DIVERSITY OF BIRD POPULATIONS AT PATLAKHAWA PROTECTED FOREST, COOCHBEHAR, WEST BENGAL, INDIA
}

\author{
Asitava Chatterjee, Shuvadip Adhikari, Anandamay Barik, \\ Subhra Kumar Mukhopadhyay
}

\begin{abstract}
Chatterjee A., Adhikari S., Barik A., Mukhopadhyay S.K. 2013. The mid-winter assemblage and diversity of bird populations at Patlakhawa Protected Forest, Coochbehar, West Bengal, India. Ring 35: 31-53

We compared avian community structure of six contrasting habitat types at Patlakhawa Protected Forest of Dooars of West Bengal. During three weeks of the mid-winter studies 154 bird species representing 41 families were recorded. Of the 154 recorded species, 22 were observed in grasslands, 22 in swamp forest, 46 in riverine forest, 52 at miscellaneous plantation areas, 43 at forest edges and 51 at wetlands. The Shannon-Wiener general diversity indices varied among the habitats and ranged from 2.323 to 3.458 . Richness (5.813-11.410), evenness (0.406-0.641) and dominance (0.042-0.128) indices also varied considerably among the study locations. The highest diversity, the lowest dominance and a very high evenness were characteristics of miscellaneous plantations. Grasslands showed highest evenness and considerably high species diversity. The lowest avian evenness and diversity were recorded in wetlands and swamp forests. Insectivorous birds were most numerous (53), followed by carnivorous (40), omnivorous (29) and frugivorous (15) birds. Winter appeared to be the best time for niche occupancy at the Himalayan foothills for assemblages of either winter migrants and visitors or altitudinal and passage migrants, as about $30 \%$ of all encountered species were migrants. 96 bird species were observed to occur at any one of these habitat types showing high habitat fidelity. Mixed avian foraging assemblages that varied in species number and compositions were observed and they contain 11 to 17 species. Avifaunal niche diversity and richness in different habitats of Patlakhawa Protected Forest during mid-winter envisaged its importance from conservation point of view.
\end{abstract}

A. Chatterjee, Office of the Divisional Forest Officer, Coochbehar Division, Coochbehar-736101, West Bengal, India, E-mail: asitavachatterjee@gmail.com; S. Adhikari, School of Oceanographic Studies, Jadavpur Univesity, Kolkata-700 032, India, E-mail: shuvaadhikari90 @gmail.com; A. Barik, Ecology Research Laboratory, Department of Zoology, University of Burdwan, Burdwan-713 104, India, E-mail: anandamaybarik@yahoo.co.in; S.K. Mukhopadhyay (corresponding author), Department of Zoology, Hooghly Mohsin College, Chinsurah, Hooghly-712 101, India, E-mail: msubhro@yahoo.com.

Key words: Sub-Himalayan forests, West Bengal birds, IBA 131, NWCP, foraging guild, Palaearctic-Asian migrant species 


\section{INTRODUCTION}

The Eastern Himalayan Broadleaf Forest land is one of the few Indomalaya ecozones that is globally outstanding for both species richness and levels of endemism. Overall, this ecoregion is a biodiversity hotspot. This ecoregion also plays an important role in maintaining altitudinal connectivity between the habitat types that make up the larger Himalayan ecosystem. Several birds and mammals exhibit altitudinal seasonal migrations and depend on contiguous habitat up and down the steep Himalayan slopes for unhindered movements. If any of the habitat layers, from the productive habitats in the Terai-Dooars savanna and grasslands and tropical dry forests along the foothills through the broadleaf forests and conifers to the alpine meadows in the high mountains, is lost or degraded, these processes will be disrupted. Precipitation (about $3000 \mathrm{~mm}$ ), topography, and temperature combine to influence the flora and fauna across this ecoregion. This region harbours varied forest types and such variation in vegetation has attracted a variety of bird species; a number of lotic habitats and lakes, pools and marshes are an added advantage to study the water dependant birds of this region. The Patlakhawa Protected Forest comes under Assam Plains Endemic Bird Area (EBA 131) adjacent to Jaldapara National Park and is within $15 \mathrm{~km}$ from Buxa National Park, second Tiger Reserve of West Bengal, after the Sundarbans. Both Jaldapara and Buxa are Important Bird Areas (IBAs IN 320 and IN 317, respectively). This EBA harbours a substantial number of more widespread threatened species, with high priority for conservation due to major habitat destruction and incomplete ornithological knowledge. This EBA is virtually enclosed by the mountains of the Eastern Himalayas (EBA 130) and there is a slight overlap in the altitudinal ranges of birds from these two areas (Stattersfield et al. 1998, Islam and Rahmani 2004, BirdLife International 2012a). Noteworthy recent works on the avifauna of these areas are by Crosby (1996), Allen et al. (1996), Inskipp et al. (2001), Pawar and Birand (2001), Sivakumar and Prakash (2004), Sivakumar et al. (2004, 2006), Aysegul and Samraat (2004), Naaz et al. (2005), Ahmed and Yahya (2010), Datta (2011) and Roy et al. (2011a). This is the first attempt to compare the avian diversity and community structure of different habitats at Patlakhawa Protected Forest in reference to avian foraging guilds.

\section{MATERIAL AND METHODS}

\section{Description of the study sites}

The present study was conducted in different habitats of Patlakhawa Protected Forest, situated in Sub-Himalayan Terai Region (Biogeographic Zone 7B) and flood plain of Torsha River in Coochbehar District at the north-eastern corner of West Bengal $\left(26^{\circ} 26^{\prime}\right.$ to $26^{\circ} 29^{\prime} \mathrm{N} ; 89^{\circ} 19^{\prime}$ to $\left.89^{\circ} 21^{\prime} \mathrm{E}\right)$. The vegetation matrix of Patlakhawa Protected Forest contains Savannah woodland with intermittent grasslands. The TeraiDooars savanna and grasslands is a narrow lowland ecoregion of Indo-Malayan eco- 
zone harbouring Himalayan subtropical broadleaf forest biome at the base of the Himalayas, about $25 \mathrm{~km}$ wide and a continuation of the Gangetic Plain colloquially called Terai in the Ganges Basin east to Nepal, then Dooars in West Bengal, Bangladesh, Bhutan and Assam east to the Brahmaputra River. World's tallest grass species at the grasslands are found in this ecoregion, which are the most threatened and rare worldwide. However, a substantial area has recently been planted with miscellaneous indigenous plant species, particularly in the denuded and degraded areas. In addition to Rasomati Beel, a wetland area within Patlakhawa Protected Forest, there is a good number of perennial lotic and lentic water sources. The present study identified the prominent habitat patches depending on contrastingly varied physiographic conditions and including diverse vegetation compositions. Following habitats (Fig. 1) were selected for the present study, viz., Grasslands: correspond to Champion and Seth's (1968) 'Eastern Wet Alluvial Grassland' (4D/2D2), extending for around 150 ha; Swamp Forests: correspond to 'Tropical hill valley degraded Ardisian Swamp-forest' (4C/FS2) and covering around 100 ha; Riverine Forests: correspond partly to Champion and Seth's northern dry deciduous seral-khair-sisso association (5B/1S2), extending along the 'char' areas of the bank of Torsha river and covering around 250 ha; Miscellaneous Plantations: including both young and mature artificial plantations with indigenous plant species suitable to local environmental condition within Patlakhawa Protected Forest areas and covering nearly 800 ha; Forest Edges: including distinctly different patches located in the transition of any two covering around 10 ha; and Wetlands: including Rasomati Beel and Torsha river area and Buri Torsha, Sil Torsha and Mora Torsha rivulets. The wetland areas cover 50-60 ha depending upon inundations and water flow regime of Torsha River. Rasomati Beel has been identified as a designated wetland under National Wetland Conservation Programme (NWCP)

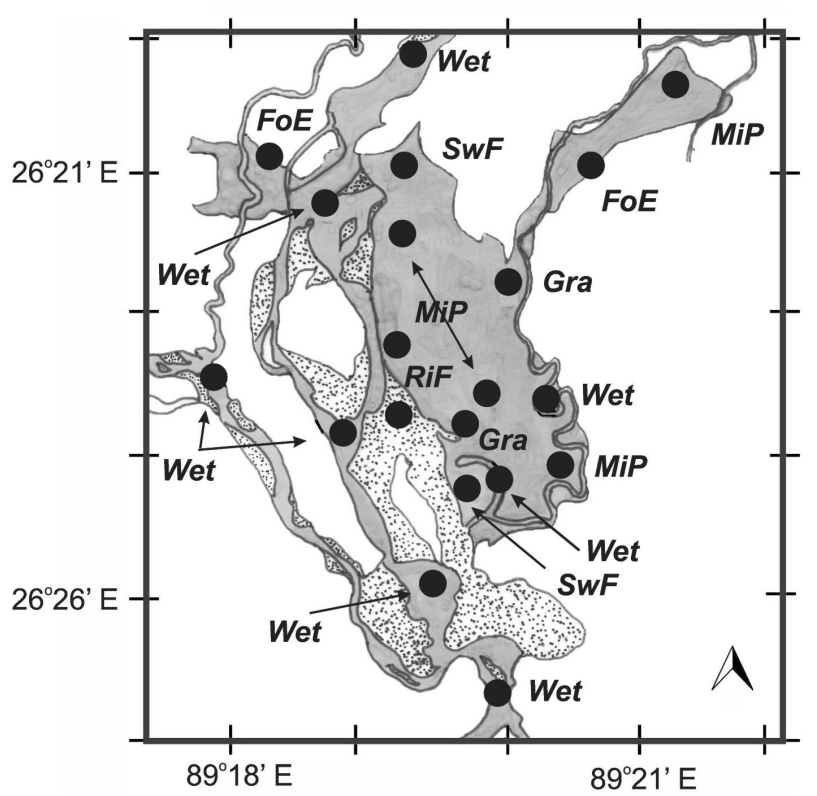

Fig. 1. Map of Patlakhawa Protected Forest, showing analysed habitats 
of Conservation and Survey Division, MoEF, Govt. of India in 2009 and these wetlands are situated in Assam-Sylhet plains Wetland Region (W14) recognized by the Kumar et at. (2005) for threatened bird species.

A three-week long study was conducted in January 2012. This period appears to be excellent to study altitudinal migrants, passage migrants and winter visitors along with the resident bird species of the region. During this mid-winter period in northBengal weather condition were stable. Average climatic conditions are reported to be highly humid with abundant rains and moderately hot summer and cold winter. Mean air temperature ranges between $10.4^{\circ} \mathrm{C}$ and $24.1^{\circ} \mathrm{C}$. The average annual rainfall is recorded as $3201.3 \mathrm{~mm}$ with $70 \%$ of annual rainfall received during south-west monsoon. On an average, the concerned study area experiences 102 raining days per year having rainfall of $2.5 \mathrm{~mm}$ or more per day. The area is with flat topography and below 10 percent slope with an altitude below $125 \mathrm{~m} \mathrm{MSL}$.

\section{Estimation of avian density except waterfowl}

In the present study, each habitat was divided into $100 \mathrm{~m}^{2}$ grids and five randomly chosen blocks, $200 \mathrm{~m}$ apart from each other to avoid overlap in recording the data, were used for point counts. Five points from each habitat were chosen from the selected grids. A total of 135 point counts at each habitat type (3 counts at each point in each day for 3 consecutive days at 5 points) during the first three hours after sunrise (5:30-8:30 h), during noon (11:30-14:30 h) and in the evening (16:30-19:30 h) were carried out. The results were given as the number of birds per hectare (numbers ha ${ }^{-1}$ ). A fixed radius circular-plot method was used to record the detections and to calculate the density of the avian species (Hutto et al. 1986, Bibby et al. 2000, Raman 2003, Sutherland 2006) at the selected habitat patches. At each point three kinds of data within a specified time period $(10 \mathrm{~min})$ were recorded: firstly, the number of individuals of each species detected within a $30-\mathrm{m}$ radius surrounding the observer and secondly, the abundance of individuals of each species detected beyond the 30-m radius but still within the habitats of interest. Finally, the species identity of individuals was carefully detected while the observer walked between count points to have a complete species list for the concerned habitat patch within our study duration. Birds that originally were detected outside the 30 -m radius boundary but that later moved and were observed within $30 \mathrm{~m}$ from the observer were recorded as occurring within the fixed-radius circle. As it was assumed that all birds within $30 \mathrm{~m}$ were detected, the census would facilitate comparisons among different habitats. Birds were detected immediately upon arrival at the count area and we continued to record them for $10 \mathrm{~min}$. All birds seen (perched or flying under the canopy) or calls heard were recorded. At each point we also noted (1) sex, (2) height of perching/flying and (3) number of individuals per flocks/parties.

\section{Estimation of waterfowl density}

We used the line transect method to record the waterfowl species richness and abundance (Hutto et al. 1986, Bibby et al. 1992, Buckland et al. 1993) at wetland areas. Methodology was described in detail in our previous publication (Roy et al. 2011b). 
The order of sampling was random; however, each of the 4 sides of the lentic body or along the side of the lotic habitats was traversed during each sampling time. We surveyed each side by walking along a transect and counted all birds seen within $50 \mathrm{~m}$ of the transect. All sections of the transect were at least $50 \mathrm{~m}$ from the edge of the shoreline. We included birds observed in the distance which was over $50 \mathrm{~m}$ in front or behind as long as they were recorded within $50 \mathrm{~m}$ perpendicular to the transect. We recorded the time and weather conditions at the start of each sampling. We recorded birds flying over the habitat separately from those using the habitat. For a morerobust estimate of the populations, we also conducted random hand-frame and binocular-frame counts (Gopal 1995) of birds in 3 selected distance ranges of 50, 100, and $150 \mathrm{~m}$. We standardized areas of both the hand-frame and binocular-frame by averaging 3 measurements and worked out the ground cover on land at preset distances. Such frame-counts encompassed all avian species, either resting on the bank or islands, wading at the edge or swimming on the water surface. We averaged 3 individual counts at 3 time intervals to obtain representative data of a particular count (Gibbons et al.1996).

\section{Systematics, migratory status and feeding guilds}

For identification, nomenclature and sequence of the families in the text, Grimmett et al. (1998) was followed in general. However, both Grimmett et al. (1998) and Kazmierczak and van Perlo (2000) were followed for identification work and common and scientific names. Foraging groups were classified as insectivorous, phytophaguscarnivorous, phytophagus, frugivorous, nectarivorous, grainivorous, carnivorous and omnivorous on the basis of feeding and foraging habits of the bird species according to our observation and as described by Ali and Ripley (1987). Mixed species foraging parties and avian assemblages on flowering trees were observed for an uninterrupted period of 30 minutes to record the number of species as well as number of individuals per species, particularly in Swamp Forests, Riverine Forests, Miscellaneous Plantations and Forest Edges habitats.

The migratory status of the birds recorded during present investigation was ascertained following Ali and Ripley (1987), Grimmett et al. (1998) and Kazmierczak and Perlo (2000). Residents (R), lived in the region throughout the year along with other migratory bird species during winter. Different migrants species those visited the forest from distant places, even outside the sub-continent, during mid-winter period were designated as the winter visitors (WV) while winter migrants (WM) generally traveled relatively shorter distances from their Himalayan breeding areas, within the continent, to their wintering sites for exploiting the conducive environment like better resources (long daylight, abundant food). The local migrants (LM), migrated between small geographic area while passage migrants (PM), on the other hand, migrated covering long distances and selected some appropriate places as stopover sites along the course of their journey. The altitudinal migrants (AM), migrated short distances along the altitudinal gradients and thereby, migration ranged between the higher reaches and the foothills during winter to avoid high altitude freezing cold and shortage of food supply. 


\section{Statistical analysis}

The Shannon-Wiener index of general diversity, Buzas and Gibson's evenness index, Margalef's richness index, Simpson's dominance index, Sorensen's similarity index, Jaccard's similarity index and Jackknife estimate were calculated to analyze the avian community structure using Past Version 2.07 and Dindex Version 4.0. For hierarchical cluster analysis to comment on the relation between the studied habitats, a dendrogram was constructed using Statistica Version 5.1A. We used SPSS for Windows, version 13.0 for analysis of variance (ANOVA) between habitat patches.

\section{RESULTS}

\section{Avian community structure}

During this short term study, 154 bird species representing 41 families were recorded within six different habitats. Bird densities recorded at different habitats and their migratory status are given in Table 1. In the present study, out of 41 families, family Corvidae showed the highest number of species (20), followed by Sylviidae (13), Anatidae (11), Passeridae (10), Muscicapidae (9), Accipitridae (6) and Ardeidae (6).

In habitat-wise occurrence (Tables 1-2), the highest number of species was recorded in Miscellaneous Plantations, followed by Wetlands, Riverine Forests-and Forest Edges. The lowest number of species was recorded in Grasslands and Swamp Forests. The highest number of birds per hectare was observed in Miscellaneous Plantations (87.4 individuals/ha), while the lowest in Grasslands (20.3 ind./ha). In Miscellaneous Plantations, 20 families were recorded and considerably high bird densities were noted in Corvidae (21.2 ind./ha), Pycnonotidae (14.7 ind./ha) and Sturnidae (10.9 ind./ha). Bird densities of other 17 families in Miscellaneous Plantations were below 10 ind./ha. In Riverine Forests, an overall bird density of 81.8 ind./ha was noted and the maximum density was for Corvidae (17.2 ind./ha) followed by Sturnidae (15.2 ind./ha) and Pycnonotidae (11.8 ind./ha). Forest Edges and Wetlands also showed high and comparable bird densities (85.2 ind./ha and 84.5 ind./ha, respectively). Important families in Forest Edges were Sturnidae (23.6 ind./ha), Sylviidae (20.1 ind./ha) and Corvidae (8.3 ind./ha). In Wetlands, important families were Dedrocygnidae (22.9 ind./ha), Anatidae (16.2 ind./ha) and Jacanidae (11.4 ind./ha). Bird densities in Swamp Forests and Grasslands were much lower (37.1 ind./ha and 20.3 ind./ha, respectively). In Swamp Forests, important families were Pycnonotidae (13.5 ind./ha), Muscicapidae (7.1 ind./ha) and Sylviidae (4.6 ind./ha); while in Grasslands these were Passeridae (4.5 ind./ha), Corvidae (3.2 ind./ha) and Cisticolidae (2.9 ind./ha) (Tables 1-2).

Density of the Red-whiskered Bulbul, Pycnonotus jocosus in Miscellaneous Plantations was the highest (7.8 ind./ha), followed by Bronzed Drongo, Dicrurus aeneus and Red-vented Bulbul, Pycnonotus cafer (both have a density of $6.8 \mathrm{ind}$./ha) and Asian Pied Starling, Sturnus contra (6.2 ind./ha). In Riverine Forests, Red-vented Bulbul occurred in maximum number (11.4 ind./ha), followed by Indian Rollar, Coracius benghalensis (6.8 ind./ha) and Cheastnut-tailed Starling, Sturnus malabaricus (6.3 ind./ha). 
Common Babbler, Turdoides caudatus (13.8 ind./ha) and Jungle Myna, Acridotheres fuscus (12.8 ind./ha) were abundant in Forest Edges. The Wetlands were dominated by Lesser Whistling-ducks, Dendrocygna javanica (22.9 ind./ha), followed by Bronzedwin- ged Jacana, Metopidius indicus (10.7 ind./ha) and Common Teal, Anas crecca (6.8 ind./ha). In Swamp Forests, important species are Red-whiskered Bulbul (8.6 ind./ha), Grey-headed Canary Flycatcher, Culicicapa ceylonensis (6.4 ind./ha) and Red-vented Bulbul (4.6 ind./ha). Important species of Grasslands are Plain Prinia, Prinia inornata (2.9 ind./ha), Great Tit, Parus major (2.6 ind./ha) and Scaly-breasted Munia, Lonchura punctulata (2.6 ind./ha).

Table 1

Checklist of bird species, foraging guilds, migratory status and habitat wise abundance (numbers/ha) in Patlakhawa Protected Forest. Foraging guild: In - Insectivorous, $\boldsymbol{P h}$ - $\boldsymbol{C}$ - Phytophagus-Carnivorous, $\boldsymbol{P h}$ - Phytophagus, $\boldsymbol{F g}$ - Frugivorous, $\boldsymbol{N} \boldsymbol{c}$ - Nectarivorous, $\boldsymbol{G} \boldsymbol{r}$ - Grainivorous, $\boldsymbol{C r}$ - Carnivorous, $\boldsymbol{O} \boldsymbol{m}$ - Omnivorous; Migratory status: $\boldsymbol{R}$ - Resident, $\boldsymbol{W} \boldsymbol{V}$ - Winter visitor, $\boldsymbol{W M}$ - Winter migrant, $\boldsymbol{L} \boldsymbol{M}$ - Local migrant, $\boldsymbol{P} \boldsymbol{M}$ - Passage migrant, $\boldsymbol{A} \boldsymbol{M}$ - Altitudinal migrant; Habitats studied: $\boldsymbol{G} \boldsymbol{r a}$ - Grasslands, $\boldsymbol{S} \boldsymbol{w} \boldsymbol{F}$ - Swamp Forests, $\boldsymbol{R} \boldsymbol{i} \boldsymbol{F}$ - Riverine Forests, $\boldsymbol{M i P}$ - Miscellaneous Plantations, $\boldsymbol{F o E}$ - Forest edges, Wet - Wetlands.

\begin{tabular}{|c|c|c|c|c|c|c|c|c|}
\hline \multirow{2}{*}{ Family/Species name } & \multirow{2}{*}{$\begin{array}{l}\text { Fora- } \\
\text { ging } \\
\text { guild }\end{array}$} & \multirow{2}{*}{\begin{tabular}{|l} 
Migra- \\
tory \\
status
\end{tabular}} & \multicolumn{6}{|c|}{ Species abundance (ind./ha) } \\
\hline & & & Gra & $S w F$ & $\boldsymbol{R i F}$ & MiP & $F o E$ & Wet \\
\hline \multicolumn{3}{|l|}{ Phasianidae } & 0.00 & 0.00 & 0.00 & 0.49 & 0.86 & 0.00 \\
\hline Gallus gallus & $\mathrm{Om}$ & $\mathrm{R}$ & 0.00 & 0.00 & 0.00 & 0.49 & 0.86 & 0.00 \\
\hline \multicolumn{3}{|l|}{ Dendrocygnidae } & 0.00 & 0.00 & 0.00 & 0.00 & 0.00 & 22.92 \\
\hline Dendrocygna javanica & $\mathrm{Ph}-\mathrm{Cr}$ & $\mathrm{R}$ & 0.00 & 0.00 & 0.00 & 0.00 & 0.00 & 22.92 \\
\hline \multicolumn{3}{|l|}{ Anatidae } & 0.00 & 0.00 & 0.00 & 0.00 & 0.00 & 16.23 \\
\hline Tadorna ferruginea & $\mathrm{Om}$ & WV & 0.00 & 0.00 & 0.00 & 0.00 & 0.00 & 1.67 \\
\hline Nettapus coromandelianus & $\mathrm{Ph}-\mathrm{Cr}$ & $\mathrm{R}$ & 0.00 & 0.00 & 0.00 & 0.00 & 0.00 & 1.80 \\
\hline Anas strepera & $\mathrm{Ph}-\mathrm{Cr}$ & WV & 0.00 & 0.00 & 0.00 & 0.00 & 0.00 & 0.72 \\
\hline Anas platyrhynchos & $\mathrm{Ph}-\mathrm{Cr}$ & WV & 0.00 & 0.00 & 0.00 & 0.00 & 0.00 & 0.52 \\
\hline Anas acuta & $\mathrm{Ph}-\mathrm{Cr}$ & WV & 0.00 & 0.00 & 0.00 & 0.00 & 0.00 & 0.42 \\
\hline Anas crecca & $\mathrm{Ph}$ & WV & 0.00 & 0.00 & 0.00 & 0.00 & 0.00 & 6.77 \\
\hline Aythya ferina & $\mathrm{Ph}-\mathrm{Cr}$ & WV & 0.00 & 0.00 & 0.00 & 0.00 & 0.00 & 0.63 \\
\hline Rhodonessa rufina & $\mathrm{Ph}-\mathrm{Cr}$ & WV & 0.00 & 0.00 & 0.00 & 0.00 & 0.00 & 0.42 \\
\hline Aythya nyroca & $\mathrm{Ph}-\mathrm{Cr}$ & WV & 0.00 & 0.00 & 0.00 & 0.00 & 0.00 & 1.04 \\
\hline Anas penelope & $\mathrm{Ph}-\mathrm{Cr}$ & WV & 0.00 & 0.00 & 0.00 & 0.00 & 0.00 & 0.86 \\
\hline Mergus merganser & $\mathrm{Cr}$ & WV & 0.00 & 0.00 & 0.00 & 0.00 & 0.00 & 1.38 \\
\hline \multicolumn{3}{|l|}{\begin{tabular}{|l|} 
Picidae \\
\end{tabular}} & 0.00 & 0.00 & 7.05 & 6.07 & 0.00 & 0.00 \\
\hline Dendrocopos canicapillus & Om & $\mathrm{R}$ & 0.00 & 0.00 & 0.78 & 0.46 & 0.00 & 0.00 \\
\hline Dinopium benghalense & In & $\mathrm{R}$ & 0.00 & 0.00 & 4.68 & 3.28 & 0.00 & 0.00 \\
\hline Chrysocolaptes lucidus & In & $\mathrm{R}$ & 0.00 & 0.00 & 1.28 & 0.64 & 0.00 & 0.00 \\
\hline Dendrocopos macei & In & $\mathrm{R}$ & 0.00 & 0.00 & 0.00 & 1.26 & 0.00 & 0.00 \\
\hline Celeus brachyurus & In & $\mathrm{R}$ & 0.00 & 0.00 & 0.31 & 0.43 & 0.00 & 0.00 \\
\hline \multicolumn{3}{|l|}{ Megalaimidae } & 0.00 & 0.00 & 6.97 & 4.07 & 0.00 & 0.00 \\
\hline Megalaima asiatica & $\mathrm{Fg}$ & $\mathrm{R}$ & 0.00 & 0.00 & 6.28 & 3.25 & 0.00 & 0.00 \\
\hline
\end{tabular}




\begin{tabular}{|c|c|c|c|c|c|c|c|c|}
\hline \multirow{2}{*}{ Family/Species name } & \multirow{2}{*}{$\begin{array}{l}\text { Fora- } \\
\text { ging } \\
\text { guild }\end{array}$} & \multirow{2}{*}{$\begin{array}{l}\text { Migra- } \\
\text { tory } \\
\text { status }\end{array}$} & \multicolumn{6}{|c|}{ Species abundance (ind./ha) } \\
\hline & & & Gra & $S w F$ & $\boldsymbol{R i F}$ & $\mathbf{M i P}$ & FoE & Wet \\
\hline Megalaima lineata & $\mathrm{Fg}$ & $\mathrm{R}$ & 0.00 & 0.00 & 0.06 & 0.82 & 0.00 & 0.00 \\
\hline Megalaima haemacephala & $\mathrm{Fg}$ & $\mathrm{R}$ & 0.00 & 0.00 & 0.63 & 0.00 & 0.00 & 0.00 \\
\hline \multicolumn{3}{|l|}{ Upupidae } & 0.62 & 0.00 & 0.00 & 0.00 & 0.00 & 0.00 \\
\hline Upupa epops & In & $\mathrm{R}$ & 0.62 & 0.00 & 0.00 & 0.00 & 0.00 & 0.00 \\
\hline \multicolumn{3}{|l|}{ Coraciidae } & 0.00 & 0.00 & 6.84 & 3.25 & 1.23 & 0.00 \\
\hline Coracius benghalensis & $\mathrm{Cr}$ & $\mathrm{R}$ & 0.00 & 0.00 & 6.84 & 3.25 & 1.23 & 0.00 \\
\hline \multicolumn{3}{|l|}{ Alcedinidae } & 0.00 & 0.00 & 0.00 & 0.00 & 0.00 & 1.73 \\
\hline Alcedo atthis & $\mathrm{Cr}$ & $\mathrm{R}$ & 0.00 & 0.00 & 0.00 & 0.00 & 0.00 & 1.73 \\
\hline \multicolumn{3}{|l|}{ Halcyonidae } & 0.00 & 0.00 & 0.10 & 0.00 & 0.83 & 1.98 \\
\hline Halcyon smyrnensis & $\mathrm{Cr}$ & $\mathrm{R}$ & 0.00 & 0.00 & 0.00 & 0.00 & 0.50 & 1.95 \\
\hline Halcyon capensis & $\mathrm{Cr}$ & $\mathrm{R}$ & 0.00 & 0.00 & 0.10 & 0.00 & 0.33 & 0.03 \\
\hline \multicolumn{3}{|l|}{ Cerylidae } & 0.00 & 0.00 & 0.00 & 0.00 & 0.00 & 2.62 \\
\hline Ceryle rudis & $\mathrm{Cr}$ & $\mathrm{R}$ & 0.00 & 0.00 & 0.00 & 0.00 & 0.00 & 2.62 \\
\hline \multicolumn{3}{|l|}{ Meropidae } & 0.00 & 0.00 & 2.36 & 1.48 & 0.68 & 1.31 \\
\hline Merops orientalis & In & $\mathrm{R}$ & 0.00 & 0.00 & 2.36 & 1.48 & 0.68 & 0.63 \\
\hline Merops philippinus & In & $\mathrm{R}$ & 0.00 & 0.00 & 0.00 & 0.00 & 0.00 & 0.68 \\
\hline \multicolumn{3}{|l|}{ Cuculidae } & 0.00 & 0.00 & 1.28 & 0.85 & 0.63 & 0.00 \\
\hline Hierococcyx varius & $\mathrm{Om}$ & $\mathrm{R}$ & 0.00 & 0.00 & 1.28 & 0.00 & 0.00 & 0.00 \\
\hline Eudynamus scolopacea & $\mathrm{Om}$ & $\mathrm{R}$ & 0.00 & 0.00 & 0.00 & 0.85 & 0.63 & 0.00 \\
\hline \multicolumn{3}{|l|}{ Centropodidae } & 0.00 & 0.00 & 0.00 & 0.00 & 3.96 & 0.00 \\
\hline Centropus sinensis & $\mathrm{Om}$ & $\mathrm{R}$ & 0.00 & 0.00 & 0.00 & 0.00 & 3.96 & 0.00 \\
\hline \multicolumn{3}{|l|}{ Psittacidae } & 0.00 & 0.00 & 2.33 & 3.44 & 0.00 & 0.00 \\
\hline Psittacula krameri & $\mathrm{Fg}$ & $\mathrm{R}$ & 0.00 & 0.00 & 0.85 & 1.73 & 0.00 & 0.00 \\
\hline Psittacula alexandri & $\mathrm{Fg}$ & $\mathrm{R}$ & 0.00 & 0.00 & 0.86 & 0.75 & 0.00 & 0.00 \\
\hline Psittacula himalayana & $\mathrm{Fg}$ & $\mathrm{R}$ & 0.00 & 0.00 & 0.62 & 0.96 & 0.00 & 0.00 \\
\hline \multicolumn{3}{|l|}{ Apodidae } & 0.18 & 0.00 & 0.00 & 0.00 & 0.23 & 0.00 \\
\hline Cypsiurus balasiensis & In & $\mathrm{R}$ & 0.18 & 0.00 & 0.00 & 0.00 & 0.23 & 0.00 \\
\hline \multicolumn{3}{|l|}{ Strigidae } & 0.00 & 0.00 & 0.85 & 0.01 & 0.03 & 0.00 \\
\hline Glaucidium cuculoides & $\mathrm{Cr}$ & $\mathrm{R}$ & 0.00 & 0.00 & 0.85 & 0.00 & 0.00 & 0.00 \\
\hline Glaucidium radiatum & $\mathrm{Cr}$ & $\mathrm{R}$ & 0.00 & 0.00 & 0.00 & 0.01 & 0.00 & 0.00 \\
\hline Athene brama & $\mathrm{Cr}$ & $\mathrm{R}$ & 0.00 & 0.00 & 0.00 & 0.00 & 0.03 & 0.00 \\
\hline \multicolumn{3}{|l|}{ Caprimulgidae } & 0.00 & 0.00 & 0.08 & 0.12 & 0.00 & 0.00 \\
\hline Caprimulgus indicus & In & $\mathrm{R}$ & 0.00 & 0.00 & 0.08 & 0.12 & 0.00 & 0.00 \\
\hline \multicolumn{3}{|l|}{ Columbidae } & 1.38 & 0.00 & 5.65 & 4.10 & 8.12 & 0.00 \\
\hline Chalcophaps indica & $\mathrm{Fg} / \mathrm{Gr}$ & $\mathrm{R}$ & 0.00 & 0.00 & 0.00 & 0.68 & 0.00 & 0.00 \\
\hline Streptopelia chinensis & $\mathrm{Gr}$ & $\mathrm{R}$ & 1.38 & 0.00 & 2.64 & 3.02 & 3.26 & 0.00 \\
\hline Streptopelia decaocto & $\mathrm{Gr}$ & LM & 0.00 & 0.00 & 2.36 & 0.00 & 0.00 & 0.00 \\
\hline Streptopelia tranquebarica & $\mathrm{Gr}$ & $\mathrm{R}$ & 0.00 & 0.00 & 0.00 & 0.00 & 4.86 & 0.00 \\
\hline Treron phoenicoptera & $\mathrm{Fg}$ & $\mathrm{R}$ & 0.00 & 0.00 & 0.65 & 0.40 & 0.00 & 0.00 \\
\hline \multicolumn{3}{|l|}{ Rallidae } & 0.00 & 0.00 & 0.00 & 0.00 & 0.00 & 2.36 \\
\hline Amaurornis phoenicurus & $\mathrm{Om}$ & $\mathrm{R}$ & 0.00 & 0.00 & 0.00 & 0.00 & 0.00 & 0.94 \\
\hline Gallinula chloropus & $\mathrm{Om}$ & $\mathrm{R}$ & 0.00 & 0.00 & 0.00 & 0.00 & 0.00 & 1.42 \\
\hline \multicolumn{3}{|l|}{ Scolopacidae } & 0.00 & 0.00 & 0.00 & 0.00 & 0.00 & 2.08 \\
\hline
\end{tabular}




\begin{tabular}{|c|c|c|c|c|c|c|c|c|}
\hline \multirow{2}{*}{ Family/Species name } & \multirow{2}{*}{$\begin{array}{l}\text { Fora- } \\
\text { ging } \\
\text { guild }\end{array}$} & \multirow{2}{*}{\begin{tabular}{|c|} 
Migra- \\
tory \\
status \\
\end{tabular}} & \multicolumn{6}{|c|}{ Species abundance (ind./ha) } \\
\hline & & & Gra & $S w F$ & $\boldsymbol{R i F}$ & $M i P$ & $F o E$ & Wet \\
\hline Gallinago megala & $\mathrm{Cr}$ & WV & 0.00 & 0.00 & 0.00 & 0.00 & 0.00 & 0.21 \\
\hline Gallinago gallinago & $\mathrm{Cr}$ & WV & 0.00 & 0.00 & 0.00 & 0.00 & 0.00 & 0.46 \\
\hline Tringa ochropus & $\mathrm{Cr}$ & WV & 0.00 & 0.00 & 0.00 & 0.00 & 0.00 & 0.34 \\
\hline Tringa stagnatilis & $\mathrm{Cr}$ & WV & 0.00 & 0.00 & 0.00 & 0.00 & 0.00 & 0.45 \\
\hline Actitis hypoleucos & $\mathrm{Cr}$ & WV & 0.00 & 0.00 & 0.00 & 0.00 & 0.00 & 0.62 \\
\hline \multicolumn{3}{|l|}{ Jacanidae } & 0.00 & 0.00 & 0.00 & 0.00 & 0.00 & 11.45 \\
\hline Hydrophasianus chirurgus & $\mathrm{Om}$ & $\mathrm{R}$ & 0.00 & 0.00 & 0.00 & 0.00 & 0.00 & 0.70 \\
\hline Metopidius indicus & $\mathrm{Om}$ & $\mathrm{R}$ & 0.00 & 0.00 & 0.00 & 0.00 & 0.00 & 10.75 \\
\hline \multicolumn{3}{|l|}{ Charadriidae } & 0.00 & 0.00 & 0.00 & 0.00 & 0.00 & 5.97 \\
\hline Vanellus cinereus & $\mathrm{Cr}$ & WV & 0.00 & 0.00 & 0.00 & 0.00 & 0.00 & 0.83 \\
\hline Vanellus duvaucelii & $\mathrm{Cr}$ & $\mathrm{R}$ & 0.00 & 0.00 & 0.00 & 0.00 & 0.00 & 2.75 \\
\hline Vanellus vanellus & $\mathrm{Cr}$ & WV & 0.00 & 0.00 & 0.00 & 0.00 & 0.00 & 0.31 \\
\hline Vanellus indicus & $\mathrm{Om}$ & $\mathrm{R}$ & 0.00 & 0.00 & 0.00 & 0.00 & 0.00 & 1.46 \\
\hline Charadrius dubius & $\mathrm{Cr}$ & $\mathrm{R}$ & 0.00 & 0.00 & 0.00 & 0.00 & 0.00 & 0.62 \\
\hline \multicolumn{3}{|l|}{ Accipitridae } & 0.18 & 0.00 & 0.84 & 2.65 & 0.62 & 1.04 \\
\hline Pandion haliaetus & $\mathrm{Cr}$ & WV & 0.00 & 0.00 & 0.00 & 0.00 & 0.00 & 0.62 \\
\hline Milvus migrans & $\mathrm{Om}$ & $\mathrm{R}$ & 0.00 & 0.00 & 0.00 & 2.38 & 0.00 & 0.00 \\
\hline Haliastur indus & $\mathrm{Cr}$ & $\mathrm{R}$ & 0.00 & 0.00 & 0.00 & 0.00 & 0.00 & 0.42 \\
\hline Elanus caeruleus & $\mathrm{Cr}$ & $\mathrm{R}$ & 0.18 & 0.00 & 0.00 & 0.00 & 0.00 & 0.00 \\
\hline Accipiter badius & $\mathrm{Cr}$ & $\mathrm{R}$ & 0.00 & 0.00 & 0.00 & 0.00 & 0.62 & 0.00 \\
\hline Spilornis cheela & $\mathrm{Cr}$ & $\mathrm{R}$ & 0.00 & 0.00 & 0.84 & 0.27 & 0.00 & 0.00 \\
\hline \multicolumn{3}{|l|}{ Falconidae } & 0.38 & 0.00 & 0.00 & 0.00 & 0.44 & 0.00 \\
\hline Microhierax caerulescens & $\mathrm{Cr}$ & $\mathrm{R}$ & 0.00 & 0.00 & 0.00 & 0.00 & 0.12 & 0.00 \\
\hline Falco tinnunculus & $\mathrm{Cr}$ & WV & 0.38 & 0.00 & 0.00 & 0.00 & 0.00 & 0.00 \\
\hline Falco peregrinus & $\mathrm{Cr}$ & $\mathrm{R}$ & 0.00 & 0.00 & 0.00 & 0.00 & 0.32 & 0.00 \\
\hline \multicolumn{3}{|l|}{ Podicipedidae } & 0.00 & 0.00 & 0.00 & 0.00 & 0.00 & 0.64 \\
\hline Tachybaptus ruficollis & $\mathrm{Cr}$ & $\mathrm{R}$ & 0.00 & 0.00 & 0.00 & 0.00 & 0.00 & 0.64 \\
\hline Phalacrocoracidae & & & 0.00 & 0.00 & 0.00 & 0.00 & 0.00 & 1.82 \\
\hline Phalacrocorax carbo & $\mathrm{Cr}$ & $\mathrm{R}$ & 0.00 & 0.00 & 0.00 & 0.00 & 0.00 & 0.03 \\
\hline Phalacrocorax niger & $\mathrm{Cr}$ & $\mathrm{R}$ & 0.00 & 0.00 & 0.00 & 0.00 & 0.00 & 1.79 \\
\hline \multicolumn{3}{|l|}{ Ardeidae } & 0.00 & 0.00 & 0.00 & 0.00 & 1.31 & 5.36 \\
\hline Ardeola grayii & $\mathrm{Cr}$ & $\mathrm{R}$ & 0.00 & 0.00 & 0.00 & 0.00 & 0.00 & 1.53 \\
\hline Ardea cinerea & $\mathrm{Cr}$ & $\mathrm{R}$ & 0.00 & 0.00 & 0.00 & 0.00 & 0.00 & 0.23 \\
\hline Casmerodius albus & $\mathrm{Cr}$ & $\mathrm{R}$ & 0.00 & 0.00 & 0.00 & 0.00 & 0.00 & 0.21 \\
\hline Mesophoyx intermedia & $\mathrm{Cr}$ & $\mathrm{R}$ & 0.00 & 0.00 & 0.00 & 0.00 & 0.00 & 1.93 \\
\hline Egretta garzetta & $\mathrm{Cr}$ & $\mathrm{R}$ & 0.00 & 0.00 & 0.00 & 0.00 & 0.00 & 1.46 \\
\hline Bubulcus ibis & $\mathrm{Cr}$ & $\mathrm{R}$ & 0.00 & 0.00 & 0.00 & 0.00 & 1.31 & 0.00 \\
\hline \multicolumn{3}{|l|}{ Ciconiidae } & 0.00 & 0.00 & 0.00 & 0.00 & 0.00 & 2.79 \\
\hline Anastomus oscitans & $\mathrm{Cr}$ & $\mathrm{R}$ & 0.00 & 0.00 & 0.00 & 0.00 & 0.00 & 2.58 \\
\hline Leptoptilos javanicus & $\mathrm{Cr}$ & $\mathrm{R}$ & 0.00 & 0.00 & 0.00 & 0.00 & 0.00 & 0.21 \\
\hline Irenidae & & & 0.00 & 0.00 & 0.78 & 1.09 & 0.00 & 0.00 \\
\hline Chloropsis aurifrons & $\mathrm{Nc}$ & $\mathrm{R}$ & 0.00 & 0.00 & 0.78 & 0.68 & 0.00 & 0.00 \\
\hline
\end{tabular}




\begin{tabular}{|c|c|c|c|c|c|c|c|c|}
\hline \multirow{2}{*}{ Family/Species name } & \multirow{2}{*}{$\begin{array}{l}\text { Fora- } \\
\text { ging } \\
\text { guild }\end{array}$} & \multirow{2}{*}{\begin{tabular}{|c|} 
Migra- \\
tory \\
status
\end{tabular}} & \multicolumn{6}{|c|}{ Species abundance (ind./ha) } \\
\hline & & & Gra & $S w F$ & $\boldsymbol{R i F}$ & MiP & FoE & Wet \\
\hline Irena puella & $\mathrm{Om}$ & $\mathrm{R}$ & 0.00 & 0.00 & 0.00 & 0.41 & 0.00 & 0.00 \\
\hline \multicolumn{3}{|l|}{ Laniidae } & 0.68 & 0.00 & 0.34 & 2.68 & 2.48 & 0.00 \\
\hline Lanius schach & $\mathrm{Cr}$ & $\mathrm{R}$ & 0.00 & 0.00 & 0.00 & 0.00 & 1.86 & 0.00 \\
\hline Lanius vittatus & In & $\mathrm{R}$ & 0.46 & 0.00 & 0.00 & 2.68 & 0.00 & 0.00 \\
\hline Lanius isabellinus & $\mathrm{Cr}$ & WV & 0.22 & 0.00 & 0.34 & 0.00 & 0.01 & 0.00 \\
\hline Lanius cristatus & $\mathrm{Cr}$ & WV & 0.00 & 0.00 & 0.00 & 0.00 & 0.61 & 0.00 \\
\hline \multicolumn{3}{|l|}{ Corvidae } & 3.18 & 1.34 & 17.19 & 21.22 & 8.33 & 1.36 \\
\hline Corvus macrorhynchos & $\mathrm{Om}$ & $\mathrm{R}$ & 0.00 & 0.00 & 0.37 & 0.38 & 0.43 & 0.00 \\
\hline Corvus splendens & $\mathrm{Om}$ & $\mathrm{R}$ & 0.32 & 0.00 & 0.28 & 1.04 & 2.36 & 1.36 \\
\hline Oriolus xanthornus & $\mathrm{Fg}$ & $\mathrm{R}$ & 0.00 & 0.00 & 2.83 & 0.81 & 0.42 & 0.00 \\
\hline Oriolus traillii & $\mathrm{Om}$ & $\mathrm{R}$ & 0.00 & 0.00 & 0.00 & 0.06 & 0.00 & 0.00 \\
\hline Dendrocitta vagabunda & Om & $\mathrm{R}$ & 0.00 & 0.00 & 1.04 & 1.56 & 2.60 & 0.00 \\
\hline Dicrurus macrocercus & $\mathrm{Om}$ & $\mathrm{R}$ & 0.00 & 0.00 & 0.44 & 1.01 & 1.45 & 0.00 \\
\hline Dicrurus hottentottus & $\mathrm{Nc}$ & $\mathrm{R}$ & 0.00 & 0.00 & 4.84 & 2.08 & 0.00 & 0.00 \\
\hline Dicrurus paradiseus & In & $\mathrm{R}$ & 0.00 & 0.00 & 2.38 & 3.75 & 0.00 & 0.00 \\
\hline Dicrurus remifer & In & $\mathrm{R}$ & 0.00 & 0.00 & 0.63 & 0.60 & 0.00 & 0.00 \\
\hline Dicrurus aeneus & In & $\mathrm{R}$ & 0.00 & 0.00 & 2.38 & 6.85 & 0.00 & 0.00 \\
\hline Dicrurus leucophaeus & $\mathrm{Om}$ & WV & 0.00 & 0.00 & 0.00 & 0.00 & 0.65 & 0.00 \\
\hline Rhipidura albicollis & In & R/AM & 0.00 & 0.83 & 0.00 & 1.58 & 0.00 & 0.00 \\
\hline Hypothymis azurea & In & $\mathrm{R} / \mathrm{AM}$ & 0.48 & 0.51 & 0.00 & 0.00 & 0.00 & 0.00 \\
\hline Aegithina tiphia & In & $\mathrm{R}$ & 2.38 & 0.00 & 0.00 & 0.00 & 0.00 & 0.00 \\
\hline Pericrocotus flammeus & In & $\mathrm{R} / \mathrm{AM}$ & 0.00 & 0.00 & 1.28 & 0.00 & 0.00 & 0.00 \\
\hline Pericrocotus solaris & In & $\mathrm{R} / \mathrm{AM}$ & 0.00 & 0.00 & 0.39 & 0.00 & 0.00 & 0.00 \\
\hline Pericrocotus cinnamomeus & In & $\mathrm{R}$ & 0.00 & 0.00 & 0.34 & 0.26 & 0.00 & 0.00 \\
\hline Coracina melaschistos & In & $\mathrm{R}$ & 0.00 & 0.00 & 0.00 & 0.00 & 0.04 & 0.00 \\
\hline Coracina macei & In & $\mathrm{R} / \mathrm{LM}$ & 0.00 & 0.00 & 0.00 & 0.85 & 0.38 & 0.00 \\
\hline Coracina melanoptera & In & $\mathrm{R}$ & 0.00 & 0.00 & 0.00 & 0.39 & 0.00 & 0.00 \\
\hline \multicolumn{3}{|l|}{ Muscicapidae } & 1.43 & 7.06 & 0.02 & 4.67 & 6.90 & 0.26 \\
\hline Myophonus caeruleus & In & $\mathrm{R} / \mathrm{AM}$ & 0.00 & 0.00 & 0.00 & 0.00 & 0.86 & 0.00 \\
\hline Ficedula westermanni & In & WV/AM & 0.00 & 0.42 & 0.00 & 0.00 & 0.00 & 0.00 \\
\hline Culicicapa ceylonensis & In & WM & 0.00 & 6.37 & 0.00 & 2.38 & 0.00 & 0.00 \\
\hline Eumyias thalassina & In & WM & 0.00 & 0.26 & 0.00 & 0.00 & 0.14 & 0.00 \\
\hline Ficedula parva & In & WM & 0.00 & 0.01 & 0.02 & 0.00 & 0.00 & 0.00 \\
\hline Copsychus malabaricus & In & $\mathrm{R}$ & 0.00 & 0.00 & 0.00 & 1.01 & 1.64 & 0.00 \\
\hline Copsychus saularis & In & $\mathrm{R}$ & 1.43 & 0.00 & 0.00 & 1.28 & 3.21 & 0.00 \\
\hline Rhyacornis fuliginosus & In & $\mathrm{R} / \mathrm{AM}$ & 0.00 & 0.00 & 0.00 & 0.00 & 0.00 & 0.26 \\
\hline Saxicola torquata & In & WM & 0.00 & 0.00 & 0.00 & 0.00 & 1.05 & 0.00 \\
\hline \multicolumn{3}{|l|}{ Sturnidae } & 0.00 & 1.27 & 15.22 & 10.86 & 23.56 & 0.00 \\
\hline Sturnus malabaricus & $\mathrm{Om}$ & $\mathrm{R}$ & 0.00 & 0.00 & 6.29 & 0.00 & 0.00 & 0.00 \\
\hline Sturnus contra & $\mathrm{Om}$ & $\mathrm{R}$ & 0.00 & 0.00 & 3.12 & 6.16 & 6.38 & 0.00 \\
\hline Acridotheres tristis & $\mathrm{Om}$ & $\mathrm{R}$ & 0.00 & 1.27 & 2.19 & 2.02 & 4.36 & 0.00 \\
\hline Acridotheres fuscus & $\mathrm{Om}$ & $\mathrm{R}$ & 0.00 & 0.00 & 3.62 & 2.68 & 12.82 & 0.00 \\
\hline \multicolumn{3}{|l|}{ Sittidae } & 0.00 & 2.28 & 0.00 & 0.00 & 0.00 & 0.00 \\
\hline
\end{tabular}




\begin{tabular}{|c|c|c|c|c|c|c|c|c|}
\hline \multirow{2}{*}{ Family/Species name } & \multirow{2}{*}{$\begin{array}{l}\text { Fora- } \\
\text { ging } \\
\text { guild }\end{array}$} & \multirow{2}{*}{\begin{tabular}{|c|} 
Migra- \\
tory \\
status
\end{tabular}} & \multicolumn{6}{|c|}{ Species abundance (ind./ha) } \\
\hline & & & Gra & $S w F$ & $\boldsymbol{R i F}$ & MiP & FoE & Wet \\
\hline Sitta castanea & In & $\mathrm{R}$ & 0.00 & 2.28 & 0.00 & 0.00 & 0.00 & 0.00 \\
\hline \multicolumn{3}{|l|}{ Paridae } & 2.63 & 2.47 & 0.01 & 0.00 & 1.12 & 0.00 \\
\hline Parus major & In & $\mathrm{R}$ & 2.63 & 2.45 & 0.00 & 0.00 & 1.12 & 0.00 \\
\hline Cephalopyrus flammiceps & In & WM & 0.00 & 0.02 & 0.01 & 0.00 & 0.00 & 0.00 \\
\hline \multicolumn{3}{|l|}{ Pycnonotidae } & 0.00 & 13.48 & 11.85 & 14.67 & 0.00 & 0.00 \\
\hline Pycnonotus cafer & $\mathrm{Fg}$ & $\mathrm{R}$ & 0.00 & 4.57 & 11.43 & 6.85 & 0.00 & 0.00 \\
\hline Pycnonotus melanicterus & $\mathrm{Fg}$ & $\mathrm{AM} / \mathrm{WV}$ & 0.00 & 0.00 & 0.42 & 0.00 & 0.00 & 0.00 \\
\hline Pycnonotus leucogenys & $\mathrm{Fg}$ & $\mathrm{AM}$ & 0.00 & 0.28 & 0.00 & 0.00 & 0.00 & 0.00 \\
\hline Pycnonotus jocosus & $\mathrm{Fg}$ & $\mathrm{R}$ & 0.00 & 8.63 & 0.00 & 7.82 & 0.00 & 0.00 \\
\hline \multicolumn{3}{|l|}{ Cisticolidae } & 2.91 & 0.00 & 0.00 & 0.00 & 0.00 & 0.00 \\
\hline Prinia inornata & In & $\mathrm{R}$ & 2.91 & 0.00 & 0.00 & 0.00 & 0.00 & 0.00 \\
\hline \multicolumn{3}{|l|}{ Zosteropidae } & 0.00 & 3.68 & 0.00 & 1.28 & 0.00 & 0.00 \\
\hline Zosterops palpebrosus & $\mathrm{Om}$ & $\mathrm{R}$ & 0.00 & 3.68 & 0.00 & 1.28 & 0.00 & 0.00 \\
\hline \multicolumn{3}{|l|}{ Sylviidae } & 2.27 & 4.65 & 1.79 & 3.88 & 20.13 & 0.00 \\
\hline Orthotomus sutorius & In & $\mathrm{R}$ & 0.81 & 0.00 & 0.00 & 0.00 & 0.00 & 0.00 \\
\hline Turdoides striatus & $\mathrm{Om}$ & $\mathrm{R}$ & 0.56 & 0.85 & 1.75 & 3.86 & 6.26 & 0.00 \\
\hline Turdoides caudatus & $\mathrm{Om}$ & $\mathrm{R}$ & 0.00 & 0.00 & 0.00 & 0.00 & 13.85 & 0.00 \\
\hline Phylloscopus fuscatus & In & WV & 0.00 & 0.46 & 0.00 & 0.00 & 0.00 & 0.00 \\
\hline Phylloscopus affinis & In & WV & 0.00 & 0.02 & 0.00 & 0.00 & 0.00 & 0.00 \\
\hline Phylloscopus inornatus & In & WV & 0.00 & 0.02 & 0.00 & 0.00 & 0.00 & 0.00 \\
\hline Phylloscopus reguloides & In & WV/AM & 0.00 & 0.00 & 0.00 & 0.00 & 0.02 & 0.00 \\
\hline Phylloscopus trochiloides & In & WV & 0.00 & 0.00 & 0.03 & 0.00 & 0.00 & 0.00 \\
\hline Acrocephalus dumetorum & In & WV & 0.01 & 0.02 & 0.00 & 0.00 & 0.00 & 0.00 \\
\hline Phylloscopus griseolus & In & AM/WV & 0.00 & 0.00 & 0.01 & 0.00 & 0.00 & 0.00 \\
\hline Seicercus xanthoschistos & In & $\mathrm{AM}$ & 0.00 & 0.00 & 0.00 & 0.02 & 0.00 & 0.00 \\
\hline Phylloscopus collybita & In & WM & 0.89 & 0.00 & 0.00 & 0.00 & 0.00 & 0.00 \\
\hline Garrulax ruficollis & $\mathrm{Om}$ & $\mathrm{R}$ & 0.00 & 3.28 & 0.00 & 0.00 & 0.00 & 0.00 \\
\hline \multicolumn{3}{|l|}{ Nectarinidae } & 0.00 & 0.83 & 0.23 & 0.53 & 2.34 & 0.00 \\
\hline Dicaeum erythrorhynchos & $\mathrm{Nc} / \mathrm{Fg}$ & $\mathrm{R}$ & 0.00 & 0.82 & 0.00 & 0.38 & 0.00 & 0.00 \\
\hline Dicaeum concolor & $\mathrm{Nc} / \mathrm{Fg}$ & $\mathrm{R}$ & 0.00 & 0.01 & 0.00 & 0.01 & 0.00 & 0.00 \\
\hline Arachnothera magna & $\mathrm{Om}$ & $\mathrm{R}$ & 0.00 & 0.00 & 0.23 & 0.14 & 0.00 & 0.00 \\
\hline Nectarinia asiatica & $\mathrm{Nc} / \mathrm{In}$ & $\mathrm{R}$ & 0.00 & 0.00 & 0.00 & 0.00 & 2.34 & 0.00 \\
\hline \multicolumn{3}{|l|}{ Passeridae } & 4.50 & 0.00 & 0.00 & 0.00 & 1.40 & 2.58 \\
\hline Dendronanthus indicus & In & $\mathrm{PM} / \mathrm{WV}$ & 0.00 & 0.00 & 0.00 & 0.00 & 0.61 & 0.00 \\
\hline Motacilla alba & In & WV & 0.01 & 0.00 & 0.00 & 0.00 & 0.00 & 0.63 \\
\hline Motacilla maderaspatensis & Om & $\mathrm{R}$ & 0.00 & 0.00 & 0.00 & 0.00 & 0.00 & 0.86 \\
\hline Motacilla citreola & In & WV & 0.00 & 0.00 & 0.00 & 0.00 & 0.00 & 0.23 \\
\hline Motacilla flava & In & WV & 0.00 & 0.00 & 0.00 & 0.00 & 0.00 & 0.13 \\
\hline Motacilla cinerea & In & WV & 0.00 & 0.00 & 0.00 & 0.00 & 0.00 & 0.73 \\
\hline Lonchura punctulata & $\mathrm{Gr}$ & $\mathrm{R}$ & 2.58 & 0.00 & 0.00 & 0.00 & 0.00 & 0.00 \\
\hline Anthus rosetus & $\mathrm{Gr}$ & WV & 1.36 & 0.00 & 0.00 & 0.00 & 0.62 & 0.00 \\
\hline Anthus rufulus & In & $\mathrm{R}$ & 0.32 & 0.00 & 0.00 & 0.00 & 0.12 & 0.00 \\
\hline Anthus hodgsoni & In & WV & 0.23 & 0.00 & 0.00 & 0.00 & 0.05 & 0.00 \\
\hline \multicolumn{3}{|l|}{ TOTAL } & 20.34 & 37.06 & 81.79 & 87.41 & 85.20 & 84.50 \\
\hline
\end{tabular}


The highest species number estimate (Jack 1) and Shannon-Wiener general diversity index $\left(H^{\prime}\right)$ (Table 2$)$ were recorded in Miscellaneous Plantations. The value of the Shannon-Weiner general diversity index varied between 2.323 (Swamp Forests) and 3.458 (Miscellaneous Plantations) and there were no significant differences in this parameter among Riverine Forests, Forest Edges and Wetlands. The Margalef's Richness index $\left(D_{\text {Marg }}\right)$ varied between 5.813 (in Swamp Forests) and 11.410 (in Miscellaneous Plantations). Simpson's Dominance index $\left(D_{\text {Simp }}\right)$ varied between 0.042 (in Miscellaneous Plantations) and 0.128 (in Swamp Forests). The Buzas \& Gibson's Evenness index $\left(J^{\prime}\right)$ ranged between 0.406 (in Wetlands) and 0.641 (in Grasslands). The maximum similarity was observed between Miscellaneous Plantations and Riverine Forests in terms of both Sorensen's (S) and Jaccard's (J) similarity indices (Table 3), however, no similarity was observed between Wetlands and Swamp Forests as there was no common species.

Table 2

Avian community structure at Patlakhawa Protected Forest (number of species, their abundance and diversity indices are given; abbreviations as in Table 1)

\begin{tabular}{|l|c|c|c|c|c|c|}
\hline \multirow{2}{*}{} & \multicolumn{7}{|c|}{ Habitat types } \\
\cline { 2 - 8 } & Gra & SwF & $\boldsymbol{R i F}$ & $\boldsymbol{M i P}$ & $\boldsymbol{F o E}$ & Wet \\
\hline Species no. & 22 & 22 & 46 & 52 & 43 & 51 \\
\hline Genera no. & 19 & 17 & 30 & 37 & 31 & 34 \\
\hline Families no. & 12 & 09 & 20 & 20 & 20 & 18 \\
\hline Jackknife species number estimate $(J a c k 1)$ & 21.84 & 22.81 & 46.88 & 52.41 & 43.50 & 51.38 \\
\hline Individuals (ind./ha) & 20.34 & 37.06 & 81.79 & 87.41 & 85.20 & 84.50 \\
\hline Simpson's Dominance index $\left(D_{\text {Simp }}\right)$ & 0.088 & 0.128 & 0.057 & 0.042 & 0.076 & 0.105 \\
\hline $\begin{array}{l}\text { Shannon-Wiener general diversity } \\
\text { index }\left(H^{\prime}\right)\end{array}$ & 2.646 & 2.323 & 3.202 & 3.458 & 2.998 & 3.031 \\
\hline Buzas \& Gibson's Evenness index $\left(J^{\prime}\right)$ & 0.641 & 0.464 & 0.535 & 0.611 & 0.466 & 0.406 \\
\hline Margalef's Richness index $\left(D_{\text {Marg }}\right)$ & 6.971 & 5.813 & 10.220 & 11.410 & 9.449 & 11.270 \\
\hline
\end{tabular}

Table 3

Sorenson's (S) and Jaccard's (J) similarity indices calculated for different habitats in the study area (abbreviations as in Table 1)

\begin{tabular}{|l|c|c|c|c|c|c|c|c|c|c|}
\hline & \multicolumn{2}{|c|}{$\boldsymbol{S w F}$} & \multicolumn{2}{c|}{$\boldsymbol{R i F}$} & \multicolumn{2}{c|}{ MiP } & \multicolumn{2}{c|}{ FoE } & \multicolumn{2}{c|}{ Wet } \\
\cline { 2 - 11 } & $\mathrm{S}$ & $\mathrm{J}$ & $\mathrm{S}$ & $\mathrm{J}$ & $\mathrm{S}$ & $\mathrm{J}$ & $\mathrm{S}$ & $\mathrm{J}$ & $\mathrm{S}$ & $\mathrm{J}$ \\
\hline $\boldsymbol{G} \boldsymbol{r} \boldsymbol{a}$ & 0.182 & 0.100 & 0.118 & 0.063 & 0.135 & 0.072 & 0.308 & 0.182 & 0.027 & 0.014 \\
\hline SwF & & & 0.147 & 0.068 & 0.243 & 0.138 & 0.123 & 0.066 & 0.000 & 0.000 \\
\hline $\boldsymbol{R i F}$ & & & & & 0.653 & 0.485 & 0.315 & 0.187 & 0.041 & 0.021 \\
\hline $\boldsymbol{M i P}$ & & & & & & & 0.358 & 0.218 & 0.039 & 0.020 \\
\hline FoE & & & & & & & & & 0.085 & 0.044 \\
\hline
\end{tabular}

In the present study we observed 53 insectivorous, 40 carnivorous, 29 omnivorous and 15 frugivorous species, while nectarivorous and granivorous species were just a few, 5 and 6 respectively. Nine phytophagus-carnivorous and one phytophagus spe- 
cies were recorded and these were exclusively aquatic (Table 1). Four species, viz. Emerald Dove, Chalcophaps indica (frugi-/granivorous), Pale-billed Flowerpecker Dicaeum erythrorhynchos and Plain Flowerpecker Dicaeum concolor (nectari-/frugivorous), while Purple Sunbird Nectarinia asiatica (nectari/insectivorous) were observed to have dual foraging habits. Wetlands were dominated by carnivorous birds (26 species), whereas rest of the habitats were dominated by insectivorous birds. Both Miscellaneous Plantations and Riverine Forests harboured large number of insectivorous species (18 and 15, resp.), frugivorous (12 and 10, resp.) and omnivorous birds (16 and 12, resp.). Forest Edges habitat was dominated by representatives of insectivorous, carnivorous and omnivorous foraging guilds. Habitat wise occurrence of bird species of different foraging guilds is given in Figure 2.

In our present study we recorded 107 resident bird species along with 38 winter visitors, 6 winter migrants, 2 local migrants, 1 passage migrant and 12 altitudinal migrants. Habitat wise distribution of bird species representing different migratory
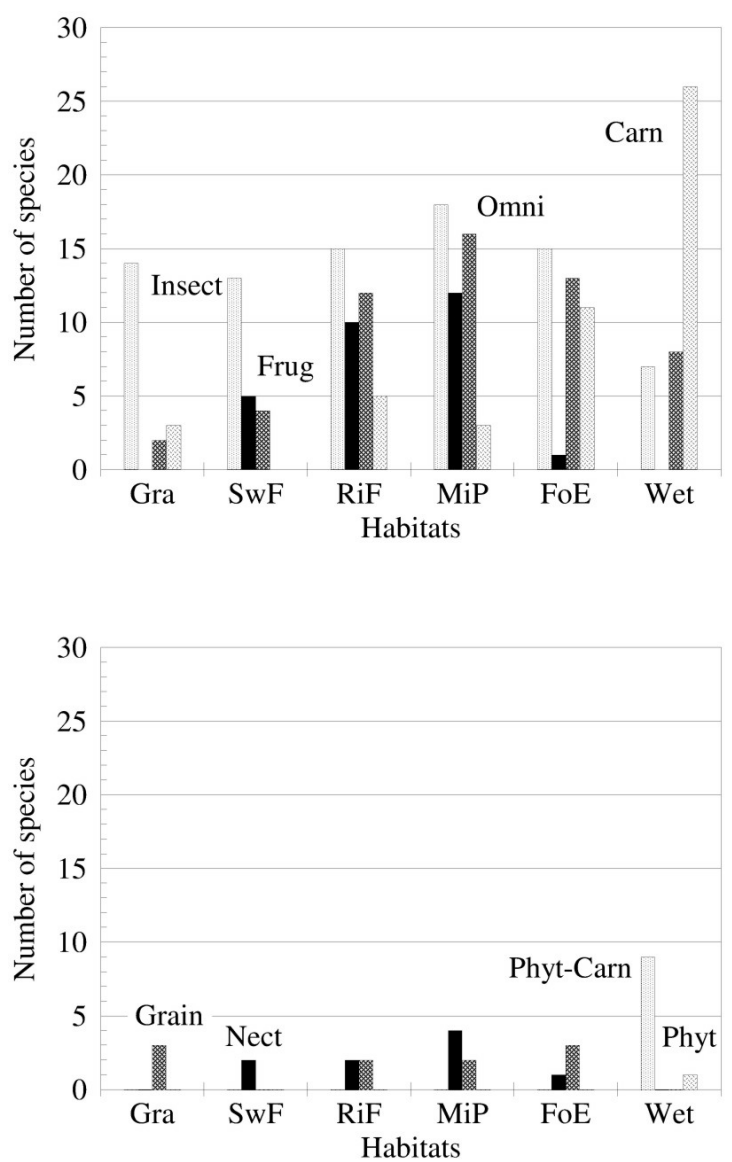

Fig. 2. Distribution of bird species of different foraging guilds, i.e. insectivorous (In), phytophagus-carnivorous ( $\mathrm{Ph}-\mathrm{Cr}$ ), frugivorous $(\mathrm{Fg})$, nectarivorous $(\mathrm{Nc})$, granivorous $(\mathrm{Gr})$, carnivorous $(\mathrm{Cr})$, omnivorous $(\mathrm{Om})$, and phytophagus $(\mathrm{Ph})$, recorded in different habitats at Patlakhawa Protected Forest. 
status is given in Figure 3. About 30\% of bird species observed during the present study was migratory in nature of different status. Local migrants species were Eurasian Collared Dove, Streptopelia decaocto and Large Cuckooshrike, Coracina macei. Forest Wagtail, Dendronanthus indicus was the only passage migrants recorded during study period at Forest Edges. Six winter migrants species belonged to families, namely, Muscicapidae (Grey-headed Canary Flycatcher; Verditer Flycatcher, Eumyias thalassina; Red-throated Flycatcher, Ficedula parva and Common Stonechat, Saxicola torquata), Paridae (Fire-capped Tit, Cephalopyrus flammiceps) and Sylviidae (Common Chiffchaff, Phylloscopus collybita). Twelve altitudinal migrants species belonged to Corvidae (White-throated Fantail, Rhipidura albicollis; Black-naped Monarch, Hypothymis azurea; Scarlet Minivet, Pericrocotus flammeus and Grey-chinned Minivet, Pericrocotus solaris), Sylviidae (Sulphur-bellied Warbler, Phylloscopus griseolus; Grey-hooded Warbler, Seicercus xanthoschistos, Blyth's Leaf Warbler, Phylloscopus reguloides), Muscicapidae (Blue Whistling Thrush, Myophonus caeruleus; Little Pied Flycatcher, Ficedula westermanni, Plumbeous Water Redstart, Rhyacornis fuliginosus) and Pycnonotidae (Black-crested Bulbul, Pycnonotus melanicterus, Hima-
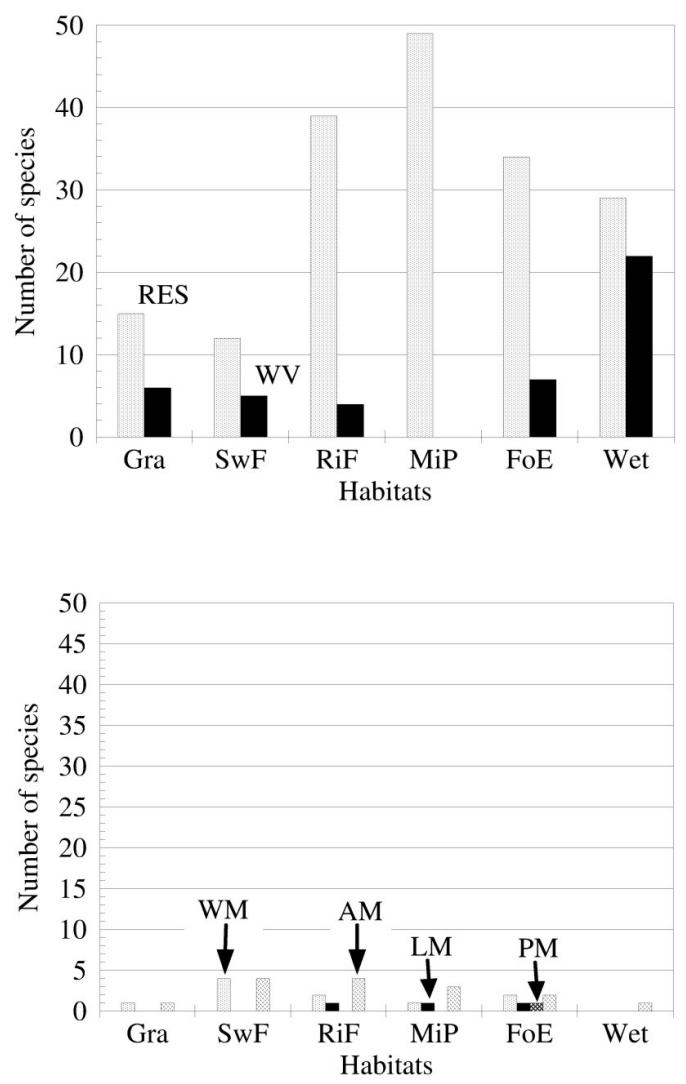

Fig. 3. Distribution of resident (RES), winter visitor (WV), winter migrant (WM), local migrant (LM), passage migrant (PM) and altitudinal migrant (AM) bird species recorded in various habitats at Patlakhawa Protected Forest. 
layan Bulbul, Pycnonotus leucogenys) families. A large number of waterbirds of the families Anatidae, Scolopacidae, Charadriidae were winter visitors in their migratory status (Table 1).

In the question of habitat fidelity, 96 bird species were observed to occur at any out of six studied habitat types, 41 species at two habitats, 12 at three, 3 at four and 2 at five different habitats (Fig. 4). However, no bird species was observed to occur at all six habitat types. Percent fidelity was calculated as the ratio between number of species present exclusively in a particular habitat and total number of species recorded from that habitat to emphasize the degree of faithfulness of avian species to a habitat. Among 51 bird species found in WL, 46 were dedicated to that habitat. In the present study, among 154 bird species, 96 were observed to occur at any one of the six habitat types investigated. WL, as obvious, showed maximum percent fidelity (90.20\%) and the minimum (15.38\%) was recorded for MP (Fig. 5). Present dataset pointed out the degrees of habitat fidelity and spectrum of specialized species in terms of habitat preference and resource utilization.

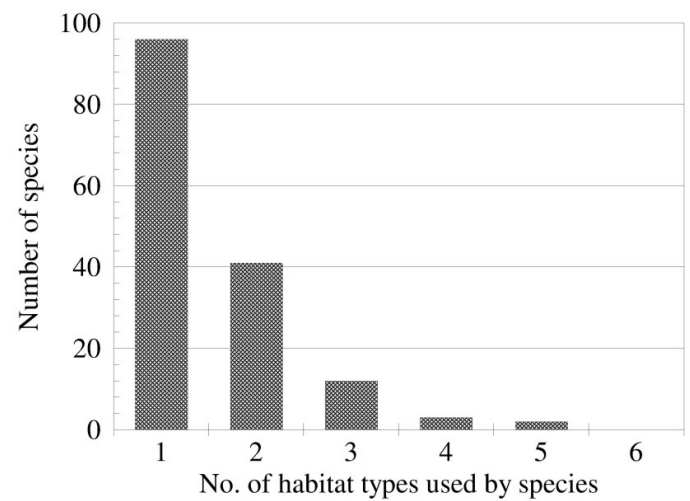

Fig. 4. Habitat fidelity of bird species at Patlakhawa Protected Forest. Number of bird species in a single habitat, and consequently 2-6 studied habitats are given in the graph.

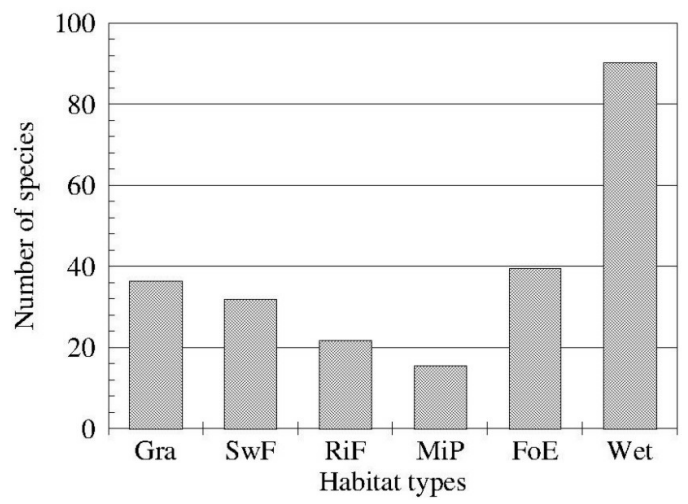

Fig. 5. Avian fidelity in different habitats (\%) at Patlakhawa Protected Forest 


\section{Mixed avian foraging assemblages}

A mixed foraging party was defined as any group of two or more birds which formation depends upon positive responses by individuals to members of their own or other species (Morse 1970). These parties vary in numbers, composition, and contribution of each species in the flock and temporality of association (Morse 1977, Powell 1989, Terborgh 1990). The formation of these flocks were explained by (1) feeding enhancement and (2) predator avoidance hypotheses (Robin and Davidar 2002). In the present study, the mixed species foraging party was comprised of 11 to 17 bird species at various habitats. Species involved in these mixed foraging parties were Black Drongo, Dicrurus macrocercus; Ashy Drongo, Dicrurus leucophaeus; Lesser Rackettailed Drongo, Dicrurus remifer; Spangled Drongo, Dicrurus hottentottus; Whitethroated Fantail; Scarlet Minivet; Black-hooded Oriole, Orious xanthornus; Goldenfronted Leafbird, Chloropsis aurifrons; Lineated Barbet, Megalaima lineata; Bluethroated Barbet, Megalaima asiatica; Small Minivet, Pericrocrotus cinnamomeus; Chestnut-bellied Nuthatch, Sitta castanea; Great Tit; Red-vented Bulbul; Blackcrested Bulbul, Pycnonotus melanicterus; Red-whiskered Bulbul, Jungle Babbler, Turdoides striatus; Blue Whistling Thrush; Grey-headed Canary Flycatcher; Streaked Spiderhunter, Arachnothera magna; Purple Sunbird; Rufous-necked Laughingthrush (Garrulax ruficollis) and Verditer Flycatcher. These flocks were observed in various habitats and involved also other species like drongos, Dicrurus spp. and bulbuls, Pycnonotus spp. as nuclear species and other being followers and associates including warblers, Phylloscopus spp. In Swamp Forests, an average number of 14.7 ( $S D \pm 0.96)$ species were observed to form mixed species foraging party. It involved on average $13.5(S D \pm 2.08)$ species in Riverine Forests. $13.7(S D \pm 1.63)$ and $13.7(S D \pm 2.21)$ average bird species are involved to form mixed foraging parties in Miscellaneous Plantations and Forest Edges respectively. Major plant species flowering during the studied period and supporting avian assemblages were Bombax ceiba, Terminalia crenulata, Terminalia myriocapra, Erythrina indica; the number of species foraging on these four tree species were 7, 6, 4 and 5 respectively.

\section{Statistical analyses}

ANOVA was applied to compare the habitats depending on the abundance of bird species (Table 4).

Significant differences $(p<0.05)$ were noted between Grasslands-Riverine Forests, Gras- slands-Miscellaneous Plantations and Swamp Forests-Miscellaneous Plantations. Hierarchical cluster analysis of the habitats showed two close comparable clusters, i.e. Gras- slands-Swamp Forests and Miscellaneous Plantations-Riverine Forests. Whereas, Forest Edges and Wetlands were well separated from those two clusters. Forest Edges showed considerable cluster distance with Grasslands and Miscellaneous Plantations-Riverine Forests clusters, as Forest Edges had similarities with all type of habitats. Wetlands, a separate cluster, were the farthest from the rest of the habitat clusters in terms of avian assemblage (Fig. 6). 
Table 4

Pair-wise comparisons using ANOVA based on estimated marginal means with the Bonferroni adjustment for multiple comparisons between the habitat patches depending on the avian abundance (significant difference are given in bold, $p<0.05$; abbreviations as in Table 1)

\begin{tabular}{|c|c|c|c|c|c|}
\hline & $S w F$ & $\boldsymbol{R i F}$ & MiP & FoE & Wet \\
\hline Gra & $\begin{array}{c}0.109 \\
( \pm 0.091)\end{array}$ & $\begin{array}{c}\mathbf{0 . 3 9 9} \\
( \pm 0.128) \\
\end{array}$ & $\begin{array}{c}\mathbf{0 . 4 3 6} \\
( \pm 0.115)\end{array}$ & $\begin{array}{c}0.421 \\
( \pm 0.150)\end{array}$ & $\begin{array}{c}0.417 \\
( \pm 0.180)\end{array}$ \\
\hline$S w F$ & & $\begin{array}{c}0.290 \\
( \pm 0.136) \\
\end{array}$ & $\begin{array}{c}\mathbf{0 . 3 2 7} \\
( \pm 0.099) \\
\end{array}$ & $\begin{array}{c}0.313 \\
( \pm 0.171) \\
\end{array}$ & $\begin{array}{c}0.308 \\
( \pm 0.197) \\
\end{array}$ \\
\hline $\boldsymbol{R i F}$ & & & $\begin{array}{c}3.649 \mathrm{E}-02 \\
( \pm 0.101)\end{array}$ & $\begin{array}{c}2.214 \mathrm{E}-02 \\
( \pm 0.174)\end{array}$ & $\begin{array}{c}1.760 \mathrm{E}-02 \\
( \pm 0.219)\end{array}$ \\
\hline$M i P$ & & & & $\begin{array}{c}1.435 \mathrm{E}-02 \\
( \pm 0.160)\end{array}$ & $\begin{array}{c}1.890 \mathrm{E}-02 \\
( \pm 0.213)\end{array}$ \\
\hline$F o E$ & & & & & $\begin{array}{c}4.545 \mathrm{E}-03 \\
( \pm 0.234)\end{array}$ \\
\hline
\end{tabular}

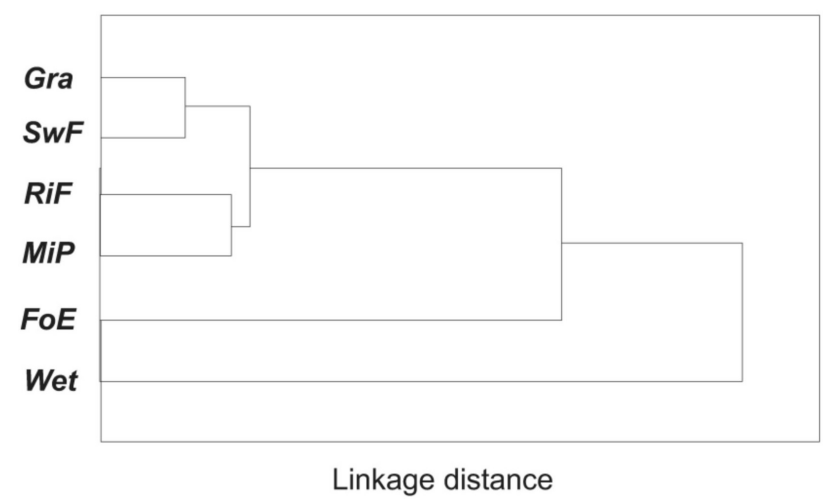

Fig. 6. Hierarchical cluster analysis of different habitats of Patlakhawa Protected Forest on the basis of avian abundance and diversity. The dendrogram was constructed using single linkage and Euclidean distances.

\section{DISCUSSION}

Conservation of forest areas of Himalayan foothills was thought to be important as these regions held different types of bird habitats. Joshi and Bhatt (2011) pointed out that in the global scale the Himalayan regions were rich in biodiversity, especially the avifauna, because of varied forest types. In the present study 154 bird species observed in six different habitat types supported their findings. Such a large number of bird species recorded in the present study, which was conducted for a short timeperiod suggest the niche diversity and richness of the region. As the present study was conducted in the mid-winter, a variety of birds that migrated to the terai-dooars forests at lower altitudes increased the avian diversity. Besides, calls of nocturnal birds were much helpful to identify them and to calculate their relative abundance in the study locations. 
Grasslands of Patlakhawa Protected Forest support a rich variety of grass species, like Phragmites karka (khagra), Imperata cylindrica (kash/thatch), Saccharum spontaneum (ikra/kash), S. narenga (dhadda), Alpinia nigra (purundi) and Arundo donax (nol). However, in the present study Grasslands supported 22 bird species which is lower compared to other habitats. Such low bird density in Grasslands might be partially attributed to short duration of survey and due to difficulty in spotting birds that took shelter inside tall grasses mainly consisting of nol, dhadda and kash. Lower visibility and occurrence of many cryptic species in grasslands could be the causes of recording lesser number of bird species in that habitat. Like Grasslands, Swamp Forests also supported 22 species, which also might be due to difficulty in accessing the swampy region and thereby the survey work was much restricted. The Swamp Forests had major plant species like Syzygium cumini, Bischofia javanica, Toona cilieta and Trewia nudiflora with a thick under-growth of Ardisia nerifolia and A. solanacae. Riverine Forests, which supported 46 bird species, was natural virgin forests along the streams, formed by different vegetation types. Major plant species of Riverine Forests were Acacia catechu, Dalbergia sissoo, Bombax ceiba, Lagerstroemia perviflora, L. reginae, Trewia nudiflora and Salix tetrasperma. Miscellaneous Plantations harboured the highest number of bird species (52) and highest value for ShannonWiener general diversity index (3.458). Mixed plantations were raised in blocks of indigenous and local miscellaneous species, which in turn resulted into a vegetation of multiple strata owing to differential growth, physiognomy and silviculture of various species. The major plant species were Acacia catechu, Dalbergia sissoo, Lagerstroemia flossreginae, Terminalia arjuna, T. belerica, T. crenulata, T. myriocapra, Phyllanthus emblica, Syzygium cumini, Bombax ceiba, Erythrina indica, Biscofia javanica, Salix tetrasperma, Dillenia indica. These mixed plantation areas, as the plants grew, took the shape of 'natural forest'. These miscellaneous plantations also supported array of undergrowth and climbers as they grew older, unlike monoculture of valuable economic species like Sal, Shorea robusta or Teak, Tectona grandis. These factors might result in diversity in food resources like insects, fruits and nectars in the miscellaneous plantation areas and consequently in diversity of bird species. Forest Edges were the habitat located in the transition between other habitat types and they might support species from both habitats and also representatives of so called edge species. In the present study we had recorded 43 species in this habitat. Vegetation structure of Riverine Forests and mature Miscellaneous Plantations areas were much alike and accordingly bird species of similar foraging behavior were observed to share these forests. Both habitats sheltered maximum representatives of insectivorous, frugivorous and omnivorous foraging guilds. Significantly, Forest Edges showed similarity with both Miscellaneous Plantations and Riverine Forests which could be attributed to use of forest edges by bird species of both habitats as a resourceful temporary foraging and resting sites owing to vegetation intergradation of two adjacent contrasting habitats. Birds of two foraging guilds, namely, phytophagus and phytophaguscarnivorous were only present in Wetlands. 51 bird species were recorded in Wetlands and they were a unique habitat for waterbirds. It seems that Wetlands completely differed from other habitats in terms of bird diversity and abundance, but the result of ANOVA did not reflect that and significant differences were observed only 
between Grasslands-Riverine Forests, Grasslands-Miscellaneous Plantations, and Swamp Forests-Miscellaneous Plantations. As the ANOVA was calculated on the basis of habitat-wise avian abundance, a high abundance was recorded in Miscellaneous Plantations, Forest Edges, Wetlands and Riverine Forests, whereas Grasslands and Swamp Forests showed considerable low abundance. Therefore, the results of hierarchical cluster analysis and ANOVA did not agree mutually. According to Thiolly et al. (1988) each forest type held its own species. The distribution and abundance of many bird species are determined by the configuration and composition of vegetation that comprised a major element of habitats (Cody 1985, Morrison 1992, Block and Brennan 1993).

Habitat-wise variations in species dominance, diversity, evenness, richness of the avian community of the terai-dooars forests during winter were interesting. Shannon-Wiener species diversity index $\left(H^{\prime}\right)$, based mainly on the proportional species abundance and reasonably independent of sample size, varied between 2.323 (in Swamp Forests) and 3.458 (in Miscellaneous Plantations). Comparable diversity values were reported by Joshi and Bhatt (2011), while working upon various habitats inside forest in Nainital District (Western Himalaya) of Uttarakhand, India. However, this general diversity index varied marginally between the habitats; almost comparable values were calculated at Riverine Forests (3.202), Wetlands (3.031) and Forest Edges (2.998) while slightly lower value was noted at Grasslands (2.646). Buzas and Gibson's index of evenness $\left(J^{\prime}\right)$ was maximum in the Grasslands where a low $H^{\prime}$ value was noted. Simpson's dominance index $\left(D_{\text {Simp }}\right)$, which was also based on proportional abundance like $H^{\prime}$, showed contrasting values to those for $H^{\prime}$. Maximum $D_{\operatorname{sim} p}$ value (0.128 for Swamp Forests) was indicating maximum dominance as the habitat was having lowest diversity as reflected by the $H^{\prime}$ value. It may be pointed out that both Shannon-Wiener and Simpson's index considered the proportional abundance of species, however, $H^{\prime}$ is more sensitive to rare species, whereas $D_{\text {Simp }}$ puts emphasis on the common species. Therefore, these indices pointed out the occurrence of many rare species in the habitats like Miscellaneous Plantations, Riverine Forests and Forest Edges reflecting lower dominance and higher diversity. A reverse situation was evident in the habitats like Swamp Forests and Grasslands suggesting lesser niche heterogeneity. Margalef's richness index $\left(D_{\text {Marg }}\right)$, which considered both abundance and species numbers, also attests the situation as the high values were associated with the habitats with high $H^{\prime}$ values while the lower values were evident in the habitats like Grasslands and Swamp Forests with lower diversity of vegetations.

In the present study we recorded one vulnerable and one near threatened species, namely, Lesser Adjutant, Leptoptilos javanicus and Ferruginous Pochard, Aythya nyroca respectively at Wetlands, however, no restricted range species were recorded at the study locations. It may be mentioned that Assam Plains EBA supported three restricted range species and adjoining Eastern Himalayas EBA harboured 22 restricted range species, out of which eight were found in West Bengal (Islam and Rahmani 2004). Among the 154 bird species two were endemic to the Indian subcontinent; viz, Jungle Babbler and White-browed Wagtail, Motacilla maderaspatensis. Three species recorded from the Patlakhawa Protected Forest, namely, Black-rumped Flameback, Dinopium benghalense; Rufous-necked Loughingthrush and Pale-billed Flowerpecker 
were designated as near-endemic to the Indian subcontinent. There were two endemic bird families, Irenidae and Megalaimidae, restricted in the Indo-malayan ecozone (Collar et al. 1994). Critically endangered species like Long-billed Vulture, Gyps indicus and White-rumped Vulture, Gyps bengalensis which had their distributions in the region remained unspotted in the present study. Likewise, representatives of families like Strigidae, Caprimulgidae, Laniidae, Muscicapidae and Sylviidae might increase significantly with long term survey works. It may be pointed out that only a few selected forest patches were studied in nearly 1650 ha Patlakhawa Protected Forest; a more intensive study involving all seasons would surely result in recording many more bird species.

Rich avifaunal diversity in the different habitats of Patlakhawa Protected Forest was surely indicative of its high importance. The Himalayan foothills are the breeding and/or resting ground of a number of Palearctic-Asian migrant bird species. However, the study was carried out in mid-winter; when we recorded a number of PalaearcticAsian winter migrants those migrate a long distance from colder boreal and temperate climate ecoregions of Palaearctic ecozone to tropical and subtropical region (Beaman 1994). Some migratory bird species from palaearctic region those mainly followed the Eurasia/south Asia or Central Asia Flyway like Dusky Warbler, Phylloscopus fuscatus (from east Palaearctic); Red-throated Flycatcher (from far west as southern Sweden and Austria); Ferruginous Pochard (widely distributed in Palaearctic and breeds in a discontinuous band from west Europe, west China, west Mongolia and Indian subcontinent); Citrine Wagtail, Motacilla citreola (from west Palaearctic) and Yellow wagtail, Motacilla flava were recorded from the present study (BirdLife International 2012b, Aich and Mukhopadhyay 2008).

This protected forest was not only important for its bird species but also supported other important wild flora and fauna. Major fauna of Patlakhawa Protected Forest included mammalian species like Sambar, Rusa unicolor; Barking deer, Muntiacus muntijak; Hog deer, Axis porcinus; Leopard, Panthera pardus; Wild Boar, Sus scrofa; Jackal, Canis aureus; Black-napped Hare, Lepus nigricollis; Jungle cat, Felis chaus; Small Indian civet, Viverricula indica. Even Asiatic Elephant, Elephas maximus and Gaur, Bos gaurus was observed to be the occasional visitor. The Patlakhawa Forest, once a hunting reserve for Maharajas of erstwhile Coochbehar state and sustained a population of Great Indian one-horned Rhinoceros, Rhinoceros unicornis till 1940s (Ahmed 1941). In 1985, last rhinoceros killing was recorded in Patlakhawa (Bahuguna and Mallick 2004). During late seventies the flood of Torsha River and human encroachments caused substantial loss to the area as well as its biological wealth. Recently, the Rasomati Wetland inside Patlakhawa Protected Forest had been identified as a designated wetland under National Wetland Conservation Programme (NWCP) of Conservation and Survey Division, MoEF, Govt. of India in 2009. It was the first attempt to protect the wetland and its habitat, which supported large verities of migratory as well as resident birds. However, such efforts were not enough to protect this forest with diverse habitat patches including a rare and pristine compact swamp-forest patch extended over 100 ha. Present workers believed that it was important to declare Patlakhawa Protected Forest as a Wildlife Sanctuary to conserve its ecosystem and species diversity. 


\section{ACKNOWLEDGMENTS}

Authors thankfully acknowledge the infrastructural support extended by the Director of Public Instruction, Govt. of West Bengal and the Hon'ble Vice Chancellor, the University of Burdwan, India. Authors also thankfully acknowledge the support, infrastructure and encouragements provided by the PCCF (HoFF), West Bengal, the Conservator of Forests, Northern Circle, West Bengal and Divisional Forest Officer, Coochbehar Division, West Bengal. They are also thankful to the forest staff and common people in and around the study areas who cooperated in many ways to complete the fieldwork. Authors are also thankful to the University Grants Commission for financial support to procure necessary instruments.

\section{LITERATURE}

Ahmad K., Yahya H.S.A. 2010. Winter diversity of birds in Makaibari Tea Estate, Kurseong, Darjeeling, India. Indian Forester 69-87.

Ahmed Y.S. 1941. A report on the Development of the Coochbehar State Forests. Coochbehar State Press.

Aich A., Mukhopadhyay S.K. 2008. Comparison of avifauna at the edges of contrasting forest patches in Western Ghat Hills of India. The Ring 30 (1/2): 71-79.

Ali S., Ripley S. D. 1987. Compact Handbook of Birds of India and Pakistan. Oxford Univ. Press.

Allen D., Anderton J., Kazmierczak K. 1996. Report on an ornithological visit to Buxa Tiger Reserve, West Bengal, India, 17 February to 6 March 1992. Forktail 12: 31-33.

Aysegul B., Samraat P. 2004. An ornithological survey in northeast India. Forktail 20: 15-24.

Bahuguna N. C., Mallick J. 2004. Ungulates of India. Wildife Institute of India. ENVIS 07(1): 1-16.

Beaman M. 1994. Palearctic birds: A checklist of the birds of Europe, North Africa and Asia north of the foothills of the Himalayas. Stonyhurst, Harrier Publications.

Bibby C.J., Burgess N.D., Hill D.A. 1992. Bird census techniques. Academic Press.

Bibby C.J., Burgess N.D., Hill D.A., Mustoe S.H. 2000. Bird Census Techniques. Academic Press.

BirdLife International. 2012a. Endemic Bird area factsheet: Assam plains. http://www.birdlife.org. Accessed on 01.09.2012.

BirdLife International. 2012b. Central Asia Factsheet. http:// www.birdlife.org. Accessed on 28.11.2012.

Block M.W., Brennan L. A. 1993. The habitat concept in ornithology. Current Ornithology 11: 35-91.

Buckland S.T., Anderson D.R., Burnham K.P., Laake J.L. 1993. Distance sampling: estimating the abundance of biological capacity of migratory shorebirds in a newly formed Zetland, Yangtze River estuary, China. Zool. Stud. 48: 769-779.

Champion H.G., Seth S.K. 1968. A Revised Survey of Forests of India. Publication Branch, Govt. of India.

Cody M.L. 1985. Habitat selection in birds. Academic Press.

Collar N.J., Cosby M.J., Stattersfield A.J. 1994. Birds to Watch 2- The World List of Threatened Birds. BirdLife International.

Crosby M. 1996. Threatened birds in the Eastern Himalayas. Oriental Bird Club Bulletin 23: 21-24. 
Datta T. 2011. Human interference and avifaunal diversity of two wetlands of Jalpaiguri, West Bengal, India. Journal of Threatened Taxa 3 (12): 2253-2262.

Gibbons D.W., Hill D., Sutherland W.J. 1996. In: Sutherland W. J. (eds). Ecological census techniques: a handbook. Cambridge Univ. Press, 27-259.

Gopal B. 1995. WWF handbook of wetland management. World Wildlife Fund (WWF) publication.

Grimmett R., Inskipp C., Inskipp T. 1998. Birds of the Indian Subcontinent. Oxford Univ. Press.

Hutto R.L., Pletschet S.M., Hendricks P. 1986. A fixed-radius point count method for nonbreeding and breeding season use. The Auk 103: 593-602.

Inskipp T., Lindsey N., Duckworth W.A. 2001. Checklist of the Birds of the Oriental Region. Oriental Bird Club.

Islam M.Z., Rahmani AR. 2004. Important Bird Area in India: Priority Sites for Conservation. IBCN, BNHS, BirdLife International.

Joshi K.K., Bhatt D. 2011. Birds of Three Different Forest (Sal, Pine and Oak) Habitats in Nainital District (Western Himalaya) of Uttarakhand, India. Nature and Science 9(7): 114-121.

Kazmierczak K., van Perlo B. 2000. A Field Guide to the Birds of India. Om Book Service.

Kumar A., Sati J.P., Tak P.C., Alfred J.R.B. 2005. Handbook on Indian Wetland Birds and their Conservation. Zoological Survey of India.

Morrison M.L. 1992. Bird abundance in forests managed for timber and wildlife resources, Biol.Conserve. 60: 127-134.

Morse D.H. 1970. Ecological aspects of some mixed species foraging flocks of birds. Ecol. Monogr. 40: $118-168$.

Morse D.H. 1977. Feeding behaviour and predator avoidance in heterospecific groups. BioScience 27: 332-329.

Naaz S., Das S.K., Mukhopadhyay S.K. 2005. Report on the ornithological visit to Terai forests of the Jalpaiguri Distict of West Bengal, India. Journal of Natural History 1(1): 79-85.

Pawar S. S., Birand A. 2001. A survey of amphibians, reptiles, and birds in Northeast India.

Powell G.V.N. 1989. On the possible contribution of the mixed species flocks to species richness in neotropical avifaunas. Behav. Ecol. Sociobiology 24: 387-393.

Raman T.R.S. 2003. Assessment of census techniques for interspecific comparisons of tropical rainforest bird densities: a field evaluation in the Western Ghats, India. Ibis 145: 9-21.

Robin V.V., Davidar P. 2002. The vertical stratification of birds in mixed species flocks at Parambiculam, South india: A comparison between habitats. Jour. Bom. Nat. Hist. Soc. 99(3): 389-399.

Roy U.S., Pal A., Banerjee P., Mukhopadhyay S.K. 2011a. Comparison of avifaunal diversity in and around Neora Valley National Park, West Bengal, India. Journal of Threatened Taxa 3(10): 2136-2142.

Roy U.S., Goswami A.R., Aich A., Mukhopadhyay S.K. 2011b. Changes in Densities of Waterbird Species in Santragachi Lake, India: Potential Changes in Limnochemical Variables. Zool. Stud. 50(1): 76-84.

Sivakumar S., Singha H., Prakash V. 2004. Notes on the population density and feeding ecology of the Collared Falconet Microhierax caerulescens in Buxa Tiger Reserve, West Bengal, India. Forktail 20: 97-99.

Sivakumar S., Prakash V. 2004. Water birds of Buxa Tiger Reserve, West Bengal. Zoos' Print Journal 19(4): 1451-1452.

Sivakumar S., Varghese J., Prakash V. 2006. Abundance of birds in different habitats in Buxa Tiger Reserve, West Bengal, India. Forktail 22: 128-133.

Stattersfield A.J., Crosby M.J., Long A.J., Wege D.C. 1998. Endemic Bird Areas of the World: Priorities for Biodiversity Conservation. BirdLife Conservation Series No. 7. BirdLife International.

Sutherland W.J. 2006. Ecological Census Techniques: A Handbook. Cambridge Univ. Press. 
Terborgh J. 1990. Mixed species flocks and polyspecific associations:costs and benefits of mixed groups to birds and monkeys. Amer. Jour. Primatol. 21: 87-100.

Thiolly J.M., Meyburg B.U. 1988. Forest fragmenation and the conservation of raptors: A survey on the Island of Java. Biol. Conserve 44: 229-250. 